\title{
Coronavirus-19: Possible Therapeutic Implications of Spironolactone and Dry Extract of Glycyrrhiza glabra L. (Licorice)
}

\author{
Decio Armanini ${ }^{1 *}$, Cristina Fiore ${ }^{1}$, Jens Bielenberg ${ }^{1 *}$, Chiara Sabbadin ${ }^{1}$ and Luciana Bordin ${ }^{2}$ \\ ${ }^{1}$ Department of Medicine-Endocrinology, University of Padua, Padua, Italy, ${ }^{2}$ Department of Molecular Medicine-Biological \\ Chemistry, University of Padua, Padua, Italy
}

https://clinicaltrials.gov/ (NCT044241349, NCT043465887, NCT04487964)

Keywords: spironolactone, licorice, androgen receptor, inflammation, plasma membrane

OPEN ACCESS

Edited by:

Paolo Montuschi,

Catholic University of the Sacred

Heart, Italy

Reviewed by:

Annalisa Trenti,

University of Padua, Italy

Anthony Ashton,

The University of Sydney, Australia

*Correspondence:

Decio Armanini

decio.armanini@unipd.it

Jens Bielenberg

jens.bielenberg@t-online.de

Specialty section: This article was submitted to Inflammation Pharmacology,

a section of the journal

Frontiers in Pharmacology

Received: 02 May 2020 Accepted: 23 September 2020

Published: 22 October 2020

Citation:

Armanini D, Fiore C, Bielenberg J, Sabbadin C and Bordin L (2020)

Coronavirus-19: Possible

Therapeutic Implications of

Spironolactone and Dry Extract of Glycyrrhiza glabra L. (Licorice).

Front. Pharmacol. 11:558418.

doi: 10.3389/fphar.2020.558418
The membrane angiotensin converting enzyme 2 (ACE2) couples to the spike protein of Coronavirus-19 (Covid-19), inducing proteolytic cleavage by a cathepsin transmembrane protease serine 2 (TMPRSS2), and fusion of the viral and cellular membranes (Hoffman et al., 2020). ACE2 is also present in a circulating soluble form that lacks the membrane anchor (Batlle et al., 2020). The virus seems to infect not only epithelial cells in the lung, but also inflammatory cells, inducing local secretion of cytokines. Co-expression of ACE2 and TMPRSS2 has frequently been found in the upper and lower respiratory tract in SARS-coronavirus target cells and could thus support viral spread in the human host and between individuals (Bertram et al., 2012).

The positive or negative implications of treatment with ACE inhibitors and angiotensin II receptor blockers (ARB) in infected hypertensive patients is the object of studies and interpretations. The spike protein of the SARS-CoV reduces ACE2 expression and worsens acute lung failure in vivo. This effect can be attenuated in experimental studies by blocking the renin-angiotensin pathway (Imai et al., 2005). A study on ACE2 knockout mice provides a molecular explanation of why SARS$\mathrm{CoV}$ infections cause severe and often lethal lung failure and suggests a rational therapy with recombinant ACE or with drugs that increase angiotensin II and ACE2 (Imai et al., 2005; Murthy et al., 2020). A possible explanation of the usefulness of therapy with drugs that increase circulating ACE 2 is that the binding of the virus to circulating ACE reduces its binding to ACE2 receptor of the cells of the tissues that possess the TMPRSS2.

In this short excursus we aim to highlight a possible positive effect on Covid-19 infections of spironolactone (SP) and of glycyrrhizin (GI), the main component of Glycyrrhiza glabra L. (licorice) root. SP increases the circulating ACE2 (Keidar et al., 2005; Kong et al., 2019; Stoll et al., 2019) and also has a powerful anti-inflammatory effect, antagonizing the inflammatory and cardiovascular effects of aldosterone even when its concentration is normal (Armanini et al., 2014). A recent study has shown that patients treated with antiandrogens for prostate cancer are protected from the coronavirus infection. This effect could be related to the blocking of TMPRSS2 (Stopsach et al., 2020). SP not only combines antialdosterone and antiandrogen activity by antagonizing both receptors, but also has a direct action in the adrenals, ovary and testes, where it locally blocks some enzymes involved in the synthesis of aldosterone and androgens. SP also has a stimulatory effect on aromatase (Armanini et al., 2014). Both these properties of SP could have a role in patients with Covid-19 infection.

A possible additive treatment with licorice could be considered, based on a report of the antiviral effect of licorice in SARS-coronavirus and in other virus diseases. In 2003, during the SARScoronavirus epidemic, Cinatl and collaborators reported that GI, the main active principle of licorice, 
TABLE 1 | SP and licorice: comparative effects.

Mechanisms of the action of SP

- Increased circulating ACE2, limiting the access of the virus to cell membranes

- Powerful anti-androgen and anti-mineralocorticoid effect with decreased expression of TMPRSS2 and decreased production of inflammatory cytokines

- Block of the membrane damage caused by MR activation in inflammatory situations

- Block of the hypertensive and hypokalemic effects of licorice

Effects of licorice actions

- Preservation of membrane integrity

- Stimulation of interferon gamma secretion

- Anti-inflammatory effect

- Partial block of TMPRSS2 due to the reduction of androgen secretion by testis, ovary and adrenals

- Control of the hypotensive and hyperkalemic effect of SP

inhibits SARS-related coronavirus replication in VERO cells in vitro. Unlike ribavirin, GI was able to reduce the absorption of the virus during the early steps of replication. A possible explanation is that GI induces nitrous oxide synthase, as virus replication was inhibited by addition of this compound (Cinatl et al., 2003).

Licorice is one of the most used medicinal plants since antiquity. Numerous ancient reports mention its use for symptoms attributable to respiratory tract infections, such as dry cough and hoarseness (Yeh et al., 2013), and recent studies have reported an antiviral effect of licorice (Fiore et al., 2008b; Hardy et al., 2012; Wang et al., 2015). GI decreases liver enzymes and improves the histological picture of chronic hepatitis C. In mice infected with Influenza virus A2, pulmonary consolidation and viral load in the lungs were significantly lower following treatment with GI than with saline. In the same model the splenic $\mathrm{T}$ cells of GI-treated mice were transferred to mice infected with the virus and all recipients survived. The authors attributed the effect to the stimulation of interferon secretion by immunocompetent cells (Utsunomiya et al., 1997). GI can inhibit HIV replication in mononuclear cell cultures of infected patients (Sasaki et al., 2002-2003). GI also has a protective effect on the plasma membrane of cells, which could be involved in its antiviral activity. A direct effect of GI on the cell membrane has been reported in human erythrocytes, where GI, in a dose-dependent manner, strengthens membrane integrity against both oxidative and proteolytic damage of band 3 membrane protein, induced in vitro (Fiore et al., 2008).

A similar protective effect is exerted by canrenone, the main metabolite of SP, in erythrocytes of patients with primary aldosteronism (Bordin et al., 2013). Based on these reports, the antiviral effect of GI seems independent of an effect on

\section{REFERENCES}

Armanini, D., Sabbadin, C., Donà, G., Clari, G., and Bordin, L. (2014). Aldosterone receptor blockers spironolactone and canrenone: two multivalent drugs. Expet Opin. Pharmacother. 15 (7), 909-912. doi:10.1517/14656566.2014.896901

Batlle, D., Wysocki, J., and Satchell, K. (2020). Soluble angiotensin-converting enzyme 2: a potential approach for coronavirus infection therapy?. Clin. Sci. (Lond.) 134 (5), 543-545. doi:10.1042/CS20200163
ACE2 in lung endothelial cells. GI could be evaluated for a possible enhancement of immune defences against virus infection and for protection of the plasma membrane from virus penetration. The main problem for the clinical use of licorice, however, is its mineralocorticoid-like effect, due to the block type 2 11-hydroxysteroid dehydrogenase and to binding of GI to mineralocorticoid receptors (Sabbadin et al., 2019). Licorice can be associated with SP and this association has already been reported in the treatment of women with polycystic ovary syndrome, where pure licorice extract (at the dose of $3.5 \mathrm{~g}$ a day) enhances the antiandrogen and antiinflammatory and thrombolytic effect of SP, hereby blocking the risk of hyperkalemia and hypotension (Sabbadin et al., 2019).

The mechanisms of action of SP and licorice are summarized in the Table 1. While this manuscript was reviewed, other papers were published (Cadegiani, 2020; Chen et al., 2020; Liaudet and Szabo, 2020; Luo et al., 2020; Murck, 2020; Zhao et al., 2020). There are some ongoing trials with SP in patients with COVID-19 disease, as reported in clinicaltrials.gov website. One is focused on changes from baseline in clinical assessment score and laboratory parameters during treatment with SP and bromexine (NCT044241349). The other is related to SP induced improvement in acute respiratory distress syndrome and in sequential organ failure assessment (NCT043465887).

Regarding to licorice there is one on-going pilot, non randomized study with licorice extract as a complementary medicine in patients with COVID-19 disease (NCT04487964, but nobody has proposed the association licorice and SP.

In conclusion, licorice does not need prescription and is available in many countries as a pure extract. We propose that the possible association of GI and SP may be considered for Covid-19 infections, taking account of the different mechanism of action of the two substances and their synergism. The additive activity of SP and licorice combination should be presented as a potential mechanism, not currently supported by evidence derived from clinical trials. Moreover, it should be mentioned that, as an extract, licorice would require a careful quantitative assessment of its active principle GI to define dose-response relationship.

\section{AUTHOR CONTRIBUTIONS}

DA, CF, JB, CS and LB conceived of the hypothesis and contributed to the draft and revision of the manuscript.

Bertram, S., Heurich, A., Lavender, H., Gierer, S., Danisch, S., Perin, P., et al. (2012). Influenza and SARS-coronavirus activating proteases TMPRSS2 and HAT are expressed at multiple sites in human respiratory and gastrointestinal tracts. PLoS One 7 (4), e35876. doi:10.1371/journal.pone.0035876

Bordin, L., Donà, G., Ragazzi, E., Andrisani, A., Ambrosini, G., Brunati, A. M., et al. (2013). Human red blood cells alterations in primary aldosteronism. J. Clin. Endocrinol. Metab. 98 (6), 2494-2501. doi:10.1210/jc.2012-3571

Cadegiani, F. A. (2020). Can spironolactone be used to prevent COVID-19induced acute respiratory distress syndrome in patients with hypertension? 
Am. J. Physiol. Endocrinol. Metab. 318 (5), E587-E588. doi:10.1152/ajpendo. 00136.2020

Chen, L., Hu, C., Hood, M., Zhang, X., Zhang, L., Kan, J., et al. (2020). A novel combination of vitamin $\mathrm{C}$, curcumin and glycyrrhizic acid potentially regulates immune and inflammatory response associated with coronavirus infections: A perspective from system biology analysis. Nutrients 12, 1193. doi:10.3390/ nu12041193

Cinatl, J., Morgenstern, B., Bauer, G., Chandra, P., Rabenau, H., and Doerr, H. W. (2003). Glycyrrhizin, an active component of liquorice roots, and replication of SARS-associated coronavirus. Lancet 361 (9374), 2045-2046. doi:10.1016/ s0140-6736(03)13615-x

Fiore, C., Bordin, L., Plellati, D., Armanini, D., and Clari, G. (2008a). Effect of glycyrrhetinic acid on membrane band 3 in human erythrocytes. Arch. Biochem. Biophys. 479, 46-51. doi:10.1016/j.abb.2008.08.011

Fiore, C., Eisenhut, M., Ragazzi, E., Pellati, D., Armanini, D., and Bielenberg, J. (2008b). Antiviral Effects of Glycyrrhiza species. Phytother. Res. 22, 141-148. doi:10.1002/ptr.22959

Hardy, M. E., Hendricks, J. M., Paulson, J. M., and Faunce, N. R. (2012). 18ßglycyrrhetinic acid inhibits rotavirus replication in culture. Virol. J. 9, 96. doi:10. 1186/1743-422X-9-96

Hoffmann, M., Kleine-Weber, H., Schroeder, S., Krüger, N., Herrler, T., Erichsen, S., et al. (2020). SARS-CoV-2 cell entry depends on ACE2 and TMPRSS2 and is blocked by a clinically proven protease inhibitor. Cell Open 181 (2), 271-280. doi:10.1016/j.cell.2020.02.052

Imai, Y., Kuba, K., Rao, S., Huan, T., Guo, F., Guan, B., et al. (2005). Angiotensinconverting enzyme 2 protects from severe acute lung failure. Nature 436 (7047), 112-116. doi:10.1038/nature03712

Keidar, S., Gamliel-Lazarovich, A., Kaplan, M., Pavlotzky, E., Hamoud, S., Hayek, T., et al. (2005). Mineralocorticoid receptor blocker increases angiotensinconverting enzyme 2 activity in congestive heart failure patients. Circ. Res. 97 (9), 946-953. doi:10.1161/CIRCRESAHA.120.317174

Ko, H. C., Wei, B. L., and Chiou, W. F. (2006) The effect of medicinal plants used in Chinese folk medicine on RANTES secretion by virus-infected human epithelial cells. J. Ethnopharmacol. 107 (2), 205-210. doi:10.1016/j.jep.2006. 03.004

Kong, E., Zhang, J., An, N., Tao, Y., Yu, W., and Wu, F. (2019). Spironolactone rescues renal dysfunction in obstructive jaundice rats by upregulating ACE2 expression. J. Cell Commun. Signal. 13 (1), 17-26. doi:10.1007/s12079-0180466-2

Liaudet, L., and Szabo, C. (2020). Blocking mineralocorticoid receptor with spironolactone may have a wide range of therapeutic actions in severe COVID-19 disease. Crit. Care 24 (1), 318. doi:10.1186/s13054-02003055-6

Luo, P., Liu, D., and Li, J. (2020). Pharmacological perspective: glycyrrhizin may be an efficacious therapeutic agent for COVID-19. Int. J. Antimicrob. Agents 55, 105995. doi:10.1016/j.ijantimicag.2020.105995
Murck, H. (2020). Symptomatic protective action of Glycyrrhizin (Licorice) in COVID-19 infection? Front. Immunol. 11, 1239. doi:10.3389/fimmu.2020.01239

Murthy, V. L., Koupenova, M., and Shah, R. V. (2020). ACEing COVID-19: a role for angiotensin axis inhibition in SARS-CoV-2 infection?. Circ. Res. 126 (12), 1682-1684. doi:10.1161/CIRCRESAHA.120.317174

Sabbadin, C., Bordin, L., Donà, G., Manso, J., Avruscio, G., and Armanini, D. (2019). Licorice: from pseudohyperaldosteronism to therapeutic uses. Front. Endocrinol. 10, 484. doi:10.3389/fendo.2019.00484

Sasaki, H., Takei, M., Kobayashi, M., Pollard, R. B., and Suzuki, F. (2002-2003). Effect of glycyrrhizin, an active component of licorice roots, on HIV replication in cultures of peripheral blood mononuclear cells from HIV-seropositive patients. Pathobiology 70 (4), 229-236. doi:10.1159/000069334

Stoll, R., Yokota, R., Sanches Aragã, D., and Casarini, D. E. (2019). Both aldosterone and spironolactone can modulate the intracellular ACE/ANG II/AT1 and ACE2/ANG (1-7)/MAS receptor axes in human mesangial cells. Physiol. Rep. 7 (11), e14105. doi:10.14814/phy2.14105

Stopsack, K. H., Mucci, L. A., Antonarakis, E., Nelson, P. S., and Kantoff, P. W. (2020). TMPRSS2 and COVID-19: serendipity or opportunity for intervention?. Cancer Discov. 10, 779-782. doi:10.1158/2159-8290.CD-20-0451

Utsunomiya, T., Kobayashi, M., Pollard, R. B., and Suzuki, F. (1997). Glycyrrhizin, an active component of licorice roots, reduces morbidity and mortality of mice infected with lethal doses of influenza virus. Antimicrob. Agents Chemother. 41 (3), 551-556. doi:10.1128/AAC.41.3.551

Wang, L., Yang, R., Yuan, B., Liu, Y., and Liu, C. (2015). The antiviral and antimicrobial activities of licorice, a widely-used Chinese herb. Acta Pharm. Sin. B 5 (4), 310-315. doi:10.1016/j.apsb.2015.05.005

Yeh, C. F., Wang, K. C., Chiang, L. C., Shieh, D. E., Yen, M. H., and Chang, J. S. (2013). Water extract of licorice had anti-viral activity against human respiratory syncytial virus in human respiratory tract cell lines. J. Ethnopharmacol. 148, 466-473. doi:10.1016/j.jep.2013.04.040

Zhao, X., Jiang, Y., Zhao, Y., Xi, H., Liu, C., Qu, F., et al. (2020). Analysis of the susceptibility to COVID-19 in pregnancy and recommendations on potential drug screening. Eur. J. Clin. Microbiol. Infect. Dis. 39 (7), 1209-1200. doi:10. 1007/s10096-020-03897-6

Conflict of Interest: The authors declare that the research was conducted in the absence of any commercial or financial relationships that could be construed as a potential conflict of interest.

Copyright $\odot 2020$ Armanini, Fiore, Bielenberg, Sabbadin and Bordin. This is an open-access article distributed under the terms of the Creative Commons Attribution License (CC BY). The use, distribution or reproduction in other forums is permitted, provided the original author(s) and the copyright owner(s) are credited and that the original publication in this journal is cited, in accordance with accepted academic practice. No use, distribution or reproduction is permitted which does not comply with these terms. 